\title{
Structural and optical characterization of titanium nitride thin films deposited by magnetron sputtering
}

\author{
Violeta Valentina MERIE ${ }^{1, a^{*}}$, Andreia MOLEA ${ }^{1, b}$, Vlad Nicolae BURNETE ${ }^{1, c}$, \\ Bogdan Viorel NEAMȚU ${ }^{1, \mathrm{~d}}$ and Gavril NEGREA ${ }^{1, \mathrm{e}}$ \\ ${ }^{1}$ Technical University of Cluj-Napoca, 103-105 Muncii Avenue, 400641, Cluj-Napoca, Romania \\ avioleta.Merie@stm.utcluj.ro, ${ }^{\text {b }}$ Andreia.Molea@chem.utcluj.ro, ${ }^{\mathrm{c}}$ \\ Nicolae.Vlad.Burnete@auto.utcluj.ro, 'Bogdan.Neamtu@stm.utcluj.ro, \\ eGavril.Negrea@ispm.utcluj.com
}

Keywords: Titanium nitride, Thin films, Magnetron sputtering, Reflectivity, Polarized, Semiconductor.

\begin{abstract}
Titanium nitride applicability covers different industries such as microelectronics, biomedicine and so on. This paper presents the analysis of the structural and optical properties of titanium nitride thin films for different deposition conditions. The samples were deposited by direct current magnetron sputtering on silicon substrates. The deposition was done at room temperature, on substrates preheated at $300{ }^{\circ} \mathrm{C}$ or on substrates that were polarized at $-40 \mathrm{~V}$ and $90 \mathrm{~V}$ respectively. The results indicate a dependency of the structural orientation with respect to the deposition process when this takes place at room temperature. When the deposition was done on a preheated substrate there was no structural orientation. A negative polarization of the substrate leads to the formation of small sized crystallites. Regarding the optical properties, the films showed good semiconductor properties and a low reflectivity.
\end{abstract}

\section{Introduction}

Titanium nitride (TiN) thin films were studied by many researchers due to their excellent properties, especially mechanical and tribological properties, corrosion resistance, wear resistance and thermodynamic stability [1-3]. Due to these properties, titanium nitride thin films can be used in a wide range of applications like: diffusion barriers for micro-electric devices, optical coatings with antireflection and antistatic properties, electrodes, biomedicine and hard coatings for tools and so on [4-9].

The most often used methods to obtain titanium nitride films are: reactive magnetron sputtering, laser ablation, ion beam deposition or plasma assisted chemical vapor deposition and so on [10-14]. The physical-chemical and mechanical/tribological properties of titanium nitride films depend on the deposition parameters. In this regard, different researches present the influence of some deposition parameters such as the deposition rate, deposition time, substrate, the heating or the polarization of the substrate on the topographical, mechanical, tribological, adhesion properties for titanium nitride thin films deposited by DC (direct current) magnetron sputtering. All the results are pointing out a change in these properties with the change in deposition parameters. A possible explanation for this change can be the growth of the deposited films after different preferential orientations.

The present paper is a study concerning the deposition of titanium nitride thin films by DC magnetron sputtering on silicon substrates at different deposition parameters (substrate 
temperature, substrate bias voltage, deposition time) and the structural and optical characterization of the obtained thin films.

\section{Materials and Methods \\ Deposition of titanium nitride thin films}

The deposition of titanium nitride films was done by direct current reactive magnetron sputtering method, using 99.99 \% purity titanium target and silicon Si (100) substrate. The experimental procedure details are presented in previously published work of the authors [4]. The parameters for titanium nitride films deposition were: (i) deposition time (10, 20 and 40 minutes) with the substrate (RT) at room temperature, (ii) deposition time (20 minutes) with the substrate at a temperature of $300{ }^{\circ} \mathrm{C}$ and (iii) deposition time (20 minutes) and polarization of the substrate at $40 \mathrm{~V}$ and $-90 \mathrm{~V}$. These studies were conducted in order to determine the influence of the deposition conditions on the structural and optical properties of titanium nitride films destined for MEMS devices applications.

\section{Characterization of titanium nitride thin films}

$\mathrm{X}$-ray diffraction analysis was carried out with an Inel Equinox 3000 diffractometer using a cobalt radiation $\left(\lambda_{1}=1.7889 \AA, \lambda_{2}=1.7928 \AA\right)$. The patterns were evaluated using JPCDS 87-0633. The mean crystallite size and lattice strain were calculated using the Williamson-Hall method. According to this method, the broadening of the diffraction line, $\beta$, is given by the sum of the broadening introduced by the crystallite size, $\beta_{\mathrm{d}}$, and the broadening introduced by the internal strain, $\beta_{\varepsilon}$ (equation (1)). These two parameters can be calculated using the equations (2) and (3) as follows [15]:

$$
\begin{aligned}
& \beta=\beta_{d}+\beta_{\varepsilon} \\
& \beta_{d}=\frac{K \cdot \lambda}{D \cdot \cos \theta} \\
& \beta_{\varepsilon}=4 \cdot \varepsilon \cdot \operatorname{tg} \theta
\end{aligned}
$$

Where $\mathrm{K}$ is Scherrer's constant, $\lambda$ is the wavelength of the X-ray radiation, $\mathrm{D}$ is the mean crystallite size, $\theta$ is the Bragg angle while $\varepsilon$ represents the internal strain. Starting from the equations mentioned above and the graphical plotting of the dependency $\beta \cdot \cos \theta=f(4 \cdot \sin \theta)$, the mean crystallite size and the internal strain can be determined. Thus, the internal strain is given by the line's slope while the mean crystallite size is given by the intersection between the line and the $\mathrm{Y}$ axis $(\beta \cdot \cos \theta)$.

$\mathrm{UV}$-Vis spectroscopy studies were conducted in order to determine the optical properties of the films. Using an aluminum mirror as reference, the diffuse reflectance spectra were measured at $8^{\circ}$ with a Lambda 35 Perkin-Elmer spectrometer equipped with an integrated sphere. The energy band gap of the titanium nitride films was determined based on the Kubelka-Munk function [16].

\section{Results and discussions}

XRD patterns of the titanium nitride films deposited at different deposition time are presented in Fig. 1. The crystalline phase of titanium nitride was identified based on JPCDS standard diffraction no. 87-0633. Based on the results obtained using the Williamson-Hall relation, it was observed that the increase in deposition time tends to decrease the structural parameters, i.e. crystallite size, lattice strain and cell volume (Table 1). It was also observed that for a deposition 
time of 10 minutes, the titanium nitride film has a textural orientation in the (111) crystalline plane, while when increasing the deposition time to 40 minutes, the preferred orientation of the deposited titanium nitride film changes to the (220) crystalline plane.



Fig. 1. XRD patterns of titanium nitride thin films deposited at different deposition time on a silicon substrate at room temperature.

The change in the preferred orientation, confirmed by other authors like [10,14, 17], can be attributed to the increased energy of the bombarding particles from titanium target. Another cause for the change in the structural parameters with the increase in the deposition time can be the change in the preferred orientation, which can deform the cell parameters and to the increased thickness of the film [10]. According to the theoretical calculation, titanium nitride films thickness increased $(0.27 \mu \mathrm{m}, 0.55 \mu \mathrm{m}$ and $1.10 \mu \mathrm{m})$ with the deposition time (10, 20 and 40 minutes). However, the change in lattice strain can be explain due to the presence of the (TiN) ${ }_{76}$ (JCPDS file no. 01-087-0626), (TiN).88 (JCPDS file no. 01-087-0630) and TiN (JCPDS file no. 01-087-0633) compounds in the structure of the deposited films. For instance, the presence of the $\mathrm{TiN}_{0.76}$ leads to the change in $2 \theta$ angle from $50.68^{\circ}$ (specific to the stoichiometric compound TiN) to $49.97^{\circ}$ in the case of the (200) crystalline plane. The values of the lattice strain given in Table 1 are the average values obtained for each film and they are influenced by the quantity of nonstoichiometric compounds. The presence of non-stoichiometric compounds was also reported by other researchers $[18,19]$.

Fig. 2 presents the XRD patterns for the titanium nitride film deposited on the silicon substrate preheated at $300{ }^{\circ} \mathrm{C}$ and the titanium nitride film deposited on the silicon substrates negatively biased at $-40 \mathrm{~V}$ and $-90 \mathrm{~V}$ respectively, when the deposition time was kept constant at 20 minutes. It is obvious that the deposition parameters have a strong influence on the chemical composition and, implicitly, on the properties of the investigated thin films. As mentioned above, nonstoichiometric compounds exist in the deposited thin films. Their existence determines a shift to the left of the peaks. We assume that the shift is more significant in the case of titanium nitride 
thin films that are containing higher quantities of non-stoichiometric compounds. When the silicon substrate was polarized at $-40 \mathrm{~V}$ and $-90 \mathrm{~V}$ for a deposition time of 20 minutes, compared to the un-polarized film, it was observed a decrease in crystallite size titanium nitride films (Table 1) from $21 \mathrm{~nm}$ for the un-polarized titanium nitride film to $16-17 \mathrm{~nm}$ for titanium nitride film for the polarized substrate. According to Benegra et al. [20] a decrease of crystallite size with the increase of negative bias can be explained by the fact that the pulsed power may not only increase the impinging atom energy, but it may also increase the ion current densities.

Table 1. Structural parameters of titanium nitride thin films

\begin{tabular}{lccc}
\hline \multicolumn{1}{c}{ Samples } & $\begin{array}{c}\text { Average } \\
\text { crystallite size } \\
{[\mathbf{n m}]}\end{array}$ & $\begin{array}{c}\text { Lattice } \\
\text { strain } \\
{[\%]}\end{array}$ & $\begin{array}{c}\text { Cell volume } \\
{\left[\mathbf{\AA}^{3}\right]}\end{array}$ \\
\hline $\mathrm{TiN} / \mathrm{Si} / 10 \mathrm{~min}$ & 37 & 0.379 & 12.73 \\
$\mathrm{TiN} / \mathrm{Si} / 20 \mathrm{~min}$ & 21 & 0.272 & 12.70 \\
$\mathrm{TiN} / \mathrm{Si} / 40 \mathrm{~min}$ & 17 & 0.160 & 12.69 \\
$\mathrm{TiN} / \mathrm{Si} / 20 \mathrm{~min} / 300{ }^{0} \mathrm{C}$ & 17 & 0.210 & 12.70 \\
$\mathrm{TiN} / \mathrm{Si} / 20 \mathrm{~min} /-40 \mathrm{~V}$ & 17 & 0.585 & 12.73 \\
$\mathrm{TiN} / \mathrm{Si} / 20 \mathrm{~min} /-90 \mathrm{~V}$ & 16 & 0.930 & 12.73 \\
$\mathrm{TiN} / \mathrm{JPCDS}$ 87-0633 & - & - & 12.73 \\
\hline
\end{tabular}

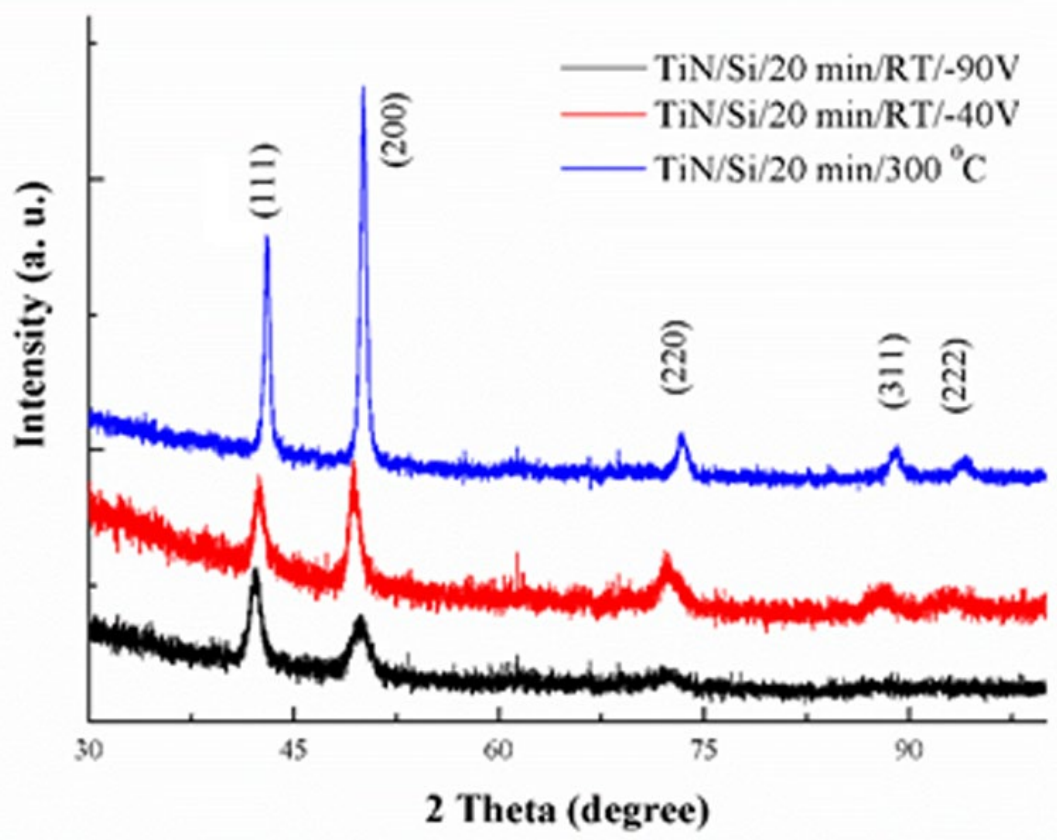

Fig. 2. XRD patterns of titanium nitride films deposited on substrates heated at a temperature 300 ${ }^{\circ} \mathrm{C}$ and polarized at $-40 \mathrm{~V}$ and $-90 \mathrm{~V}$ for 20 minutes. 
Fig. 3 shows the specular reflectance of titanium nitride films deposited on silicon substrates at room temperature at different deposition times. All the films exhibit a low reflectivity, between $300 \mathrm{~nm}$ and $500 \mathrm{~nm}$, but as the wavelength increases to the infrared region, the reflectance also increases, especially for the titanium nitride film deposited for 20 minutes which has a of reflectance nearly $60 \%$.

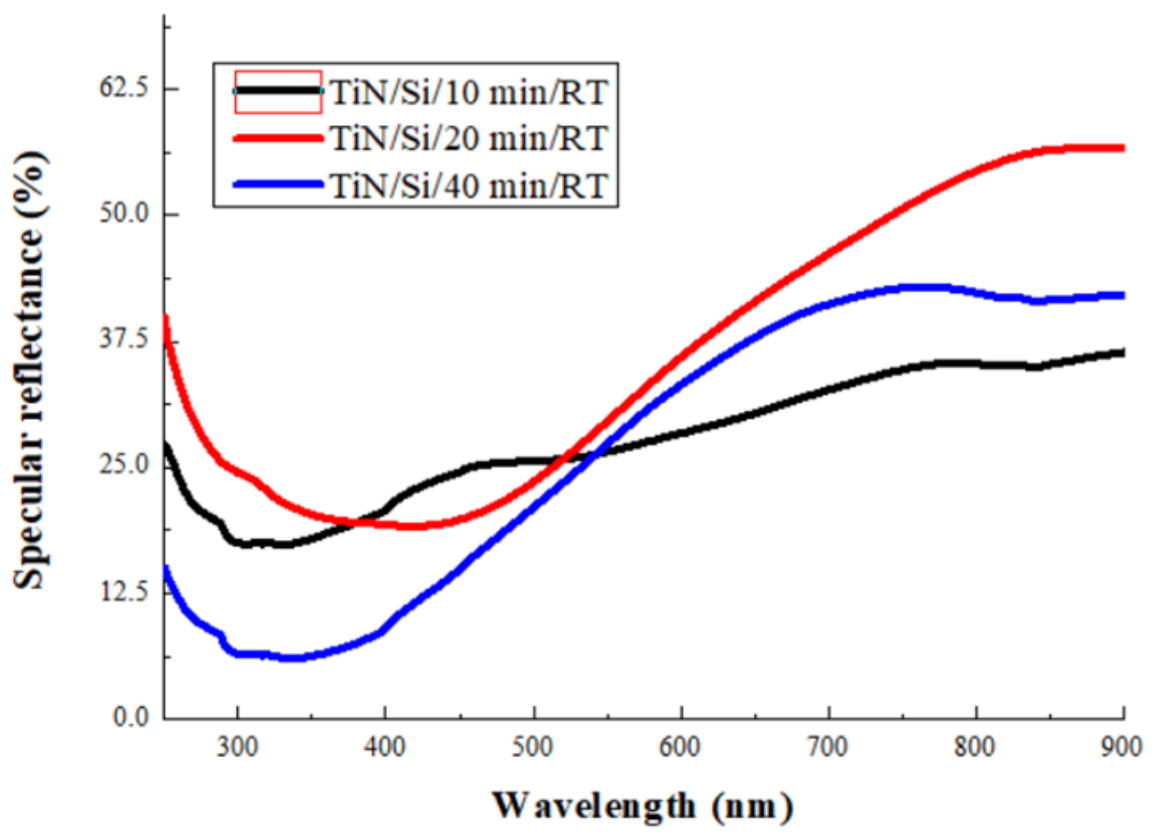

Fig. 3. Reflectance spectra of titanium nitride films deposited at different deposition time on a silicon substrate at room temperature.



Fig. 4. Energy band gap of titanium nitride films deposited at different deposition time on silicon substrate at room temperature. 
The energy band gaps suggest that the titanium nitride films deposited at different deposition times are semiconducting materials (Fig. 4). The titanium nitride film deposited for 20 minutes exhibit a lower energy band gap (2.14 eV) than the films deposited for 10 and 40 minutes, respectively (2.54 eV and $2.83 \mathrm{eV}$ ).

A red-shifting in the optical energy of titanium nitride film deposited for 20 minutes, compared to the film deposited for 10 minutes can be attributed to the increase in the film thickness and with the change in the textural orientation from (111) crystalline plane to (220) crystalline plane, which also implied an increase in the roughness of the films [4]. According to Kiran et al. [21] the change in the titanium nitride films thickness causes a shift in the optical edge and therefore a change in the band structure of the films.

Fig. 5 presents the reflectance spectra of the titanium nitride films deposited for 20 minutes on a silicon substrate heated at $300{ }^{\circ} \mathrm{C}$ and on substrates, at room temperature, to which a negative bias-voltage of $-40 \mathrm{~V}$ and $-90 \mathrm{~V}$ respectively was applied. Compared to the titanium nitride film deposited on the silicon substrate heated at $300{ }^{\circ} \mathrm{C}$, which have a reflectivity between $300 \mathrm{~nm}$ and $500 \mathrm{~nm}$, the titanium nitride films deposited on a substrate with different voltage bias, the reflectivity increases to between $500 \mathrm{~nm}$ and $900 \mathrm{~nm}$.

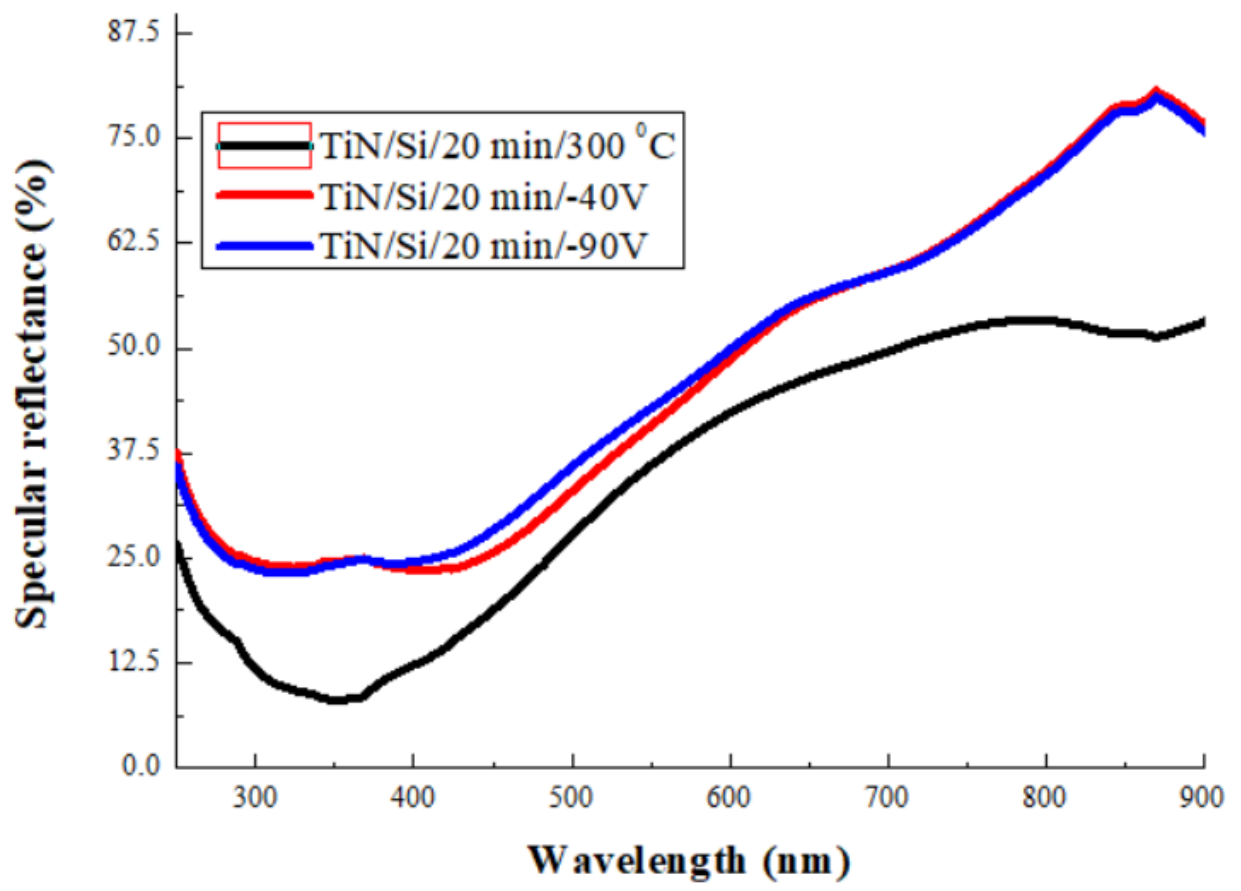

Fig. 5. Reflectance spectra of titanium nitride films deposited for 20 minutes on a silicon substrate preheated at $300{ }^{\circ} \mathrm{C}$ and deposited on a substrate at room temperature and polarized at $-40 \mathrm{~V}$ and $90 \mathrm{~V}$. 


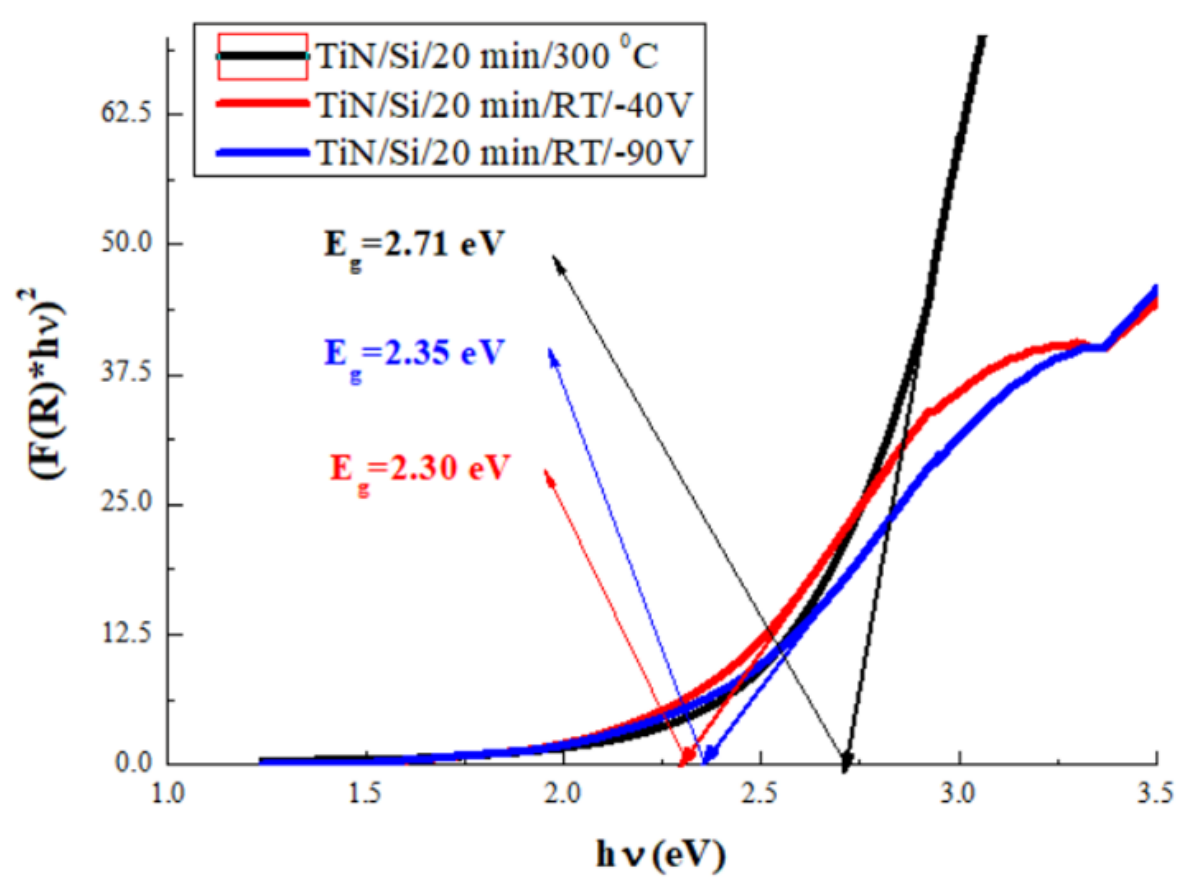

Fig. 6. Energy band gap of titanium nitride films deposited on a silicon substrate preheated at 300 ${ }^{\circ} \mathrm{C}$ and deposited on substrate at room temperature and a voltage bias of $-40 \mathrm{~V}$ and $-90 \mathrm{~V}$.

The crystalline degree of the titanium nitride film deposited on a substrate heated at $300{ }^{\circ} \mathrm{C}$ increases compared to the titanium nitride film deposited on un-heated substrate. The energy band gap (Fig. 6) also increases due to the blue-shifting of the optical response. The latter is caused by a decrease in the crystallite size that implies band structure modifications due to cell size and by the fact that titanium nitride film deposited on a heated substrate do not present a textural orientation unlike the titanium nitride film deposited on an un-heated substrate [22]. The blue-shifting of the optical response was also observed for the films deposited at negative bias voltage. This can be also attributed to the decrease in the crystallite size.

It can be concluded that titanium nitride films exhibit semiconducting properties based on the structural and optical characterization of titanium nitride films deposited on silicon substrate at different deposition conditions. As a result, titanium nitride films may have possible applications in solar cells, optoelectronics or microelectronic-mechanical devices.

\section{Summary}

The deposition of titanium nitride films on a silicon Si (100) substrate at different deposition parameters influences the structural and optical properties of the material. Thus, when the deposition time increases from 10 to 40 minutes and the deposition is done on silicon substrates at room temperature, the textural orientation changes from (111) orientation to the (220) crystalline plane due to increase in both the energy of the bombarding particles of the titanium target and in the film thickness. Heating of the silicon substrate at $300{ }^{\circ} \mathrm{C}$ leads to an increase of crystallinity, but the titanium nitride film does not present a textural orientation. Applying a negative bias voltage to the substrate leads to the formation of titanium nitride films with small crystallite size. By optical means, titanium nitride films exhibit semiconducting properties, with a low reflectivity (between $300 \mathrm{~nm}$ and $500 \mathrm{~nm}$ ), but they increase for a wavelength in the infrared region. The energy band gap of the films is also influenced by structural properties (crystallite size and textural orientation) and film thickness. 


\section{Acknowledgement}

This paper is written within the TUCN Internal Research Project Competition 2015 "Elaboration and structural, tribo-mechanical and optical characterization of some nitride thin films for MEMS applications (NitriMEMS)” (C.I.5/1.2/2015). The Internal Research Project Competition is funded by the Technical University of Cluj-Napoca in order to support and encourage young teams in addressing new topics in priority research areas of the university.

\section{References}

[1] P.J.J. Kelly, T. Vom Braucke, Z. Liu, R.D.D. Arnell, E.D.D. Doyle, Pulsed DC titanium nitride coatings for improved tribological performance and tool life (Report), Surf. Coat. Technol. 202 (2007) 774-780. https://doi.org/10.1016/j.surfcoat.2007.07.047

[2] H.Z. Durusoy, Ö. Duyar, A. Aydınlı, F. Ay, Influence of substrate temperature and bias voltage on the optical transmittance of TiN films, Vac. 70 (2003) 21-28. https://doi.org/10.1016/S0042-207X(02)00663-2

[3] K. Vasu, M.G. Krishna, K.A. Padmanabhan, Substrate-temperature dependent structure and composition variations in RF magnetron sputtered titanium nitride thin films, Appl. Surf. Sci. 257 (2011) 3069-3074. https://doi.org/10.1016/j.apsusc.2010.10.118

[4] V. Merie, M. Pustan, G. Negrea, C. Bîrleanu, Research on titanium nitride thin films deposited by reactive magnetron sputtering for MEMS applications, Appl. Surf. Sci. 358 (2015) 525-532. https://doi.org/10.1016/j.apsusc.2015.07.063

[5] X. Cao, T. Shao, S. Wen, Y. Yao, Micro/nanotribological and mechanical studies of TiN thinfilm for MEMS applications, Tribol. Trans. $47 \quad$ (2004) 227-232. https://doi.org/10.1080/05698190490439076

[6] N.Y. Kim, Y.B. Son, J.H. Oh, C.K. Hwangbo, M.C. Park, TiNx layer as an antireflection and antistatic coating for display, Surf. Coat. Technol. 128 (2000) 156-160. https://doi.org/10.1016/S0257-8972(00)00574-0

[7] D.-R. Deng, T.-H. An, Y.-J. Li, Q.-H. Wu, M.-S. Zheng, Q.-F. Dong, Hollow porous titanium nitride tubes as a cathode electrode for extremely stable Li-S batteries, J. Mater. Chem. A 4(2016) 16184-16190. https://doi.org/10.1039/C6TA07221F

[8] D. Starosvetsky, I. Gotman, Corrosion behavior of titanium nitride coated Ni-Ti shape memory surgical alloy, Biomater. 22 (2001) 1853-1859. https://doi.org/10.1016/S01429612(00)00368-9

[9] Z. Peng, H. Miao, L. Qi, S. Yang, C. Liu, Hard and wear-resistant titanium nitride coatings for cemented carbide cutting tools by pulsed high energy density plasma, Acta Mater. 51 (2003) 3085-3094. https://doi.org/10.1016/S1359-6454(03)00119-8

[10] S. Chinsakolthanakorn, A. Buranawong, S. Chiyakun, P. Limsuwan, Effects of titanium sputtering current on structure and morphology of TiZrN films prepared by reactive DC magnetron co-sputtering, Mater. Sci. Appl. $4 \quad$ (2013) 689-694. https://doi.org/10.4236/msa.2013.411086 
[11] J. Bonse, H. Sturm, D. Schmidt, W. Kautek, Chemical, morphological and accumulation phenomena in ultrashort-pulse laser ablation of TiN in air, Appl. Phys. A 71 (2000) 657-665. https://doi.org/10.1007/s003390000585

[12] A.P. Serro, C. Completo, R. Colaço, F. dos Santos, C.L. da Silva, J.M.S. Cabral, B. Saramago, A comparative study of titanium nitrides, TiN, TiNbN and TiCN, as coatings for biomedical applications, Surf. Coat. Technol. 203 (2009) 3701-3707. https://doi.org/10.1016/j.surfcoat.2009.06.010

[13] G. Zhao, C. Zhao, L. Wu, G. Duan, J. Wang, G. Han, Study on the electrical and optical properties of vanadium doped TiN thin films prepared by atmospheric pressure chemical vapor, J. Alloys Compd. 569 (2013) 1-5. https://doi.org/10.1016/j.jallcom.2013.03.110

[14] S.H. Kim, H. Park, K.H. Lee, S.H. Jee, D.-J. Kim, Y.S. Yoon, H.B. Chae, Structure and mechanical properties of titanium nitride thin films grown by reactive pulsed laser deposition, J. Ceram. Process. Res. 10 (2009) 49-53.

[15] G.K. Williamson, W.H Hall, X-ray line broadening from filed aluminum and wolfram, Acta Metallurgica 1 (1953) 22-31. https://doi.org/10.1016/0001-6160(53)90006-6

[16] A.E. Morales, E.S. Mora, U. Pal, Use of diffuse reflectance spectroscopy for optical characterization of un-supported nanostructures, Rev. Mex. Fis. 53 (2007) 18-22.

[17] A. Yazdani, M. Soltanieh, H. Aghajani, S. Rastegari, A new method for deposition of nano sized titanium nitride on steels, Vacuum $86 \quad$ (2011) 131-139. https://doi.org/10.1016/j.vacuum.2011.04.020

[18] V. Karagkiozaki, S. Logotheidis, N. Kalfagiannis, S. Lousinian, G. Giannoglou, Atomic force microscopy probing platelet activation behavior on titanium nitride nanocoatings for biomedical applications, Nanomedicine: Nanotechnology, Biology, and Medicine 5 (2009) 64-72. https://doi.org/10.1016/j.nano.2008.07.005

[19] C.-F. Hsieh, S. Jou, Titanium nitride electrodes for micro-gap discharge, Microelectronics Journal 37 (2006) 867-870. https://doi.org/10.1016/j.mejo.2006.03.003

[20] M. Benegra, D.G. Lamas, M.E. Fernández de Rapp, N. Mingolo, A.O. Kunrath, R.M. Souza, Residual stresses in titanium nitride thin films deposited by direct current and pulsed direct current unbalanced magnetron sputtering, Thin Solid Films 494 (2006)146-150. https://doi.org/10.1016/j.tsf.2005.08.214

[21] M.S.R.N. Kiran, M.G. Krishna, K.A. Padmanabhan, Growth, surface morphology, optical properties and electrical resistivity of $\varepsilon$-TiNx, Appl. Surf. Sci. 255 (2008) 1934-1941. https://doi.org/10.1016/j.apsusc.2008.06.122

[22] P. Patsalas, S. Logothetidis, Optical, electronic, and transport properties of nanocrystalline titanium nitride thin films, J. Appl. Phys. 90 (2001) 4725-4734. https://doi.org/10.1063/1.1403677 